\title{
A short daytime test using correlation dimension for respiratory movement in OSAHS
}

\author{
M. Miyata*, N. Burioka*, T. Sako*, H. Suyama*, Y. Fukuoka*, K. Tomita*, S. Higami”, E. Shimizu*
}

A short daytime test using correlation dimension for respiratory movement in OSAHS. M. Miyata, N. Burioka, T. Sako, H. Suyama, Y. Fukuoka, K. Tomita, S. Higami, E. Shimizu. C) ERS Journals Ltd 2004.

ABSTRACT: In order to examine the pathology in patients with obstructive sleep apnoea/hypopnoea syndrome (OSAHS), the nonlinear properties of respiratory movement and breath-to-breath variations during resting wakefulness with eyes closed was investigated.

Recording of the respiratory movement using inductive plethysmography was performed on 14 patients with OSAHS and 13 control subjects for $2 \mathrm{~h}$ in the supine position during daytime. To calculate the correlation dimension (D2) for respiratory movement, an algorithm proposed by Grassberger and Procaccia was applied. The indices of breath-to-breath variations were estimated. To calculate D2 and breath-tobreath variations, two different segments were selected (200 s each).

The value of $\mathrm{D} 2$ for respiratory movement in patients with OSAHS was significantly greater than that in control subjects. In the case of $\geqslant 2.0$ of D2 for respiratory movement, the sensitivity and specificity of detecting the presence of OSAHS was $85.7 \%$ and $76.9 \%$, respectively. On the basis of breath-to-breath variations, only the coefficient of variation of expiratory time for respiratory movement in patients with OSAHS was significantly greater than that in the control subjects.

In conclusion, the measurements of correlation dimensions for respiratory movement with a brief period during wakefulness may be a useful index for identifying patients with obstructive sleep apnoea/hypopnoea syndrome.

Eur Respir J 2004; 23: 885-890.
*Division of Medical Oncology and Molecular Respirology, and ${ }^{\#}$ Division of Otolaryngology, Faculty of Medicine, Tottori University, Yonago, Japan.

Correspondence: N. Burioka

Third Dept of Internal Medicine

Faculty of Medicine

Tottori University

36-1 Nishimachi

Yonago 683-8504

Japan

Fax: 81859348098

E-mail: burioka@grape.med.tottori-u.ac.jp

Keywords: Breath-to-breath variations correlation dimension

nonlinear analysis

obstructive sleep apnoea/hypopnea syndrome

Received: April 202003

Accepted after revision: January 242004
Sleep-related breathing disorders are a widespread disease, the most severe form of which is obstructive sleep apnoea/ hypopnoea syndrome (OSAHS). Sleep apnoea is generally diagnosed by polysomnography. Moreover, excessive daytime sleepiness, unrefreshing sleep, daytime fatigue and impaired concentration are principal subjective symptoms [1]. For measuring daytime sleepiness, the Epworth sleepiness scale is useful [2]. The multiple sleep latency test is widely used and is generally believed to provide a valid measurement of sleepiness on the particular day of the test [3]. However, there are few objective indicators to predict the presence of OSHAS during wakefulness.

The assessment of respiratory patterns generally proceeds from the measurement of the usual respiratory variables (e.g. tidal volume $(V \mathrm{~T})$, inspiratory $(t \mathrm{I})$ and expiratory $(t \mathrm{E})$ times, and respiratory cycle time, $(t$ tot $)$ ) on a breath-to-breath basis. PRIBAN [4] conducted the first detailed investigation into breath-to-breath variability in breathing pattern. Many investigators have utilised standard deviation (SD) or coefficients of variation $(\mathrm{CV})$ as the measure of variability of respiratory pattern $[5,6]$. However, few breath-to-breath analyses of respiration during wakefulness in patients with OSAHS have been reported. KOWALLIK et al. [7] found greater variations in nonapneic breath-to-breath intervals in patients with OSAHS than in healthy subjects, and concluded that breathing in obstructive sleep apnoea (OSA) is not only characterised by interruptions of breathing during occlusion, but by a greater variation in the pattern of normal-length breaths. However, no differences between OSA patients and healthy subjects could be detected in kurtosis during wakefulness.

Recently, nonlinear analysis has been applied to the analysis of biological time series. The correlation dimension (D2) is usually used to describe the complexity and nonlinear structure of biological signals. Recordings of electroencephalography (EEG) [8], electrocardiography (ECG) [9] and respiratory movement [10-13] reportedly have a nonlinear structure based on deterministic processes. In a previous study, the authors of the current report showed that respiratory movement in patients with severe OSAHS during apnoeic sleep was random by using nonlinear analysis [13].

The aim of the present study was to examine whether nonlinear and breath-to-breath variability analyses derived information on peculiar respiratory patterns from patients with OSAHS during wakefulness with eyes closed.

\section{Methods}

\section{Subjects}

A total of 14 males with OSAHS (age: $44 \pm 10 \mathrm{yrs}$; apnoeaplus-hypopnoea index: $39 \pm 15$ events $\cdot \mathrm{h}^{-1}$; body mass index (BMI): $29 \pm 3 \mathrm{~kg} \cdot \mathrm{m}^{-2}$ ) and 13 healthy male volunteers (age: $42 \pm 18$ yrs; BMI: $28 \pm 5 \mathrm{~kg} \cdot \mathrm{m}^{-2}$ ) participated in this study. Patients with OSAHS underwent diagnostic nocturnal polysomnography within a month before entering the study. 
Patients with lung disease, cardiac failure or cerebrovascular disease were excluded. All patients were not treated with continuous positive airway pressure and were not on some form of mediation. None of the patients had undergone surgery to treat sleep apnoea syndrome or snoring. All normal volunteers had normal sleep habits including normal sleep duration and schedule, no daytime sleepiness, no daytime nap habits, and were nonsnoring as assessed by a clinical interview and questionnaire. Informed consent was obtained from all subjects, and the university ethics committee approved the study.

\section{Experimental protocol}

Respiratory movement using inductive plethysmography was measured over a 2 -h period of resting wakefulness with eyes closed in the supine position in a dark and quiet room between 09:00 h and 12:00 h. None of the subjects had consumed alcohol or beverages containing caffeine for the $24-\mathrm{h}$ period prior to the recordings. In order to determine that the subjects remained awake during measurement, the EEG was recorded (EE2100; Nihon Electronics, Tokyo, Japan) referentially to the mastoid process at the positions $\mathrm{O} 1, \mathrm{O} 2$, F3, F4, C3 and C4 (according to the International 10-20 System, with a $60-\mathrm{Hz}$ high frequency filter and a 0.3 -s time constant). The chest bands (Respiband; NIMS Inc., North Bay Village, FL, USA) were applied to encircle the thoracic cage in order to measure respiratory movements. A total of $10 \mathrm{~min}$ were allowed for adaptation to the system before recordings were initiated. Data from the first $10 \mathrm{~min}$ were considered to represent subject adaptation and were not analysed. Movements of the thoracic cage were amplified (Respisomnography; NIMS Inc.) and recorded on magnetic tape (A-47 tape recorder; Sony, Tokyo, Japan), digitised at a sampling rate of $10 \mathrm{~Hz}$ with 12-bit resolution by an analogto-digital converter, and stored in a computer for further analysis.

\section{Data analysis}

Determination of the correlation dimension. To compute D2, intervals of 2,000 consecutive artefact-free samples (200 s) were analysed from the time series of respiratory movement of all subjects on the basis of the view of ECKMANN and RUELLE [14], who reported that a data set of at least $10^{\mathrm{D} / 2}$ in size was required for estimating a dimension. According to the opinion of ECKMANN and RUELLE [14], the intervals of 2,000 data points, as used in the present study, are acceptable for estimating a dimension. The current authors selected two different segments of the respiratory movement recordings and used the mean of the D2 values obtained from the two different segments. The value of D2 was calculated for the respiratory signals in the original data. One example of the phase space trajectories of respiratory signals is shown in figure 1 , which was constructed by three-dimensional attractors during wakefulness. A typical example from one of the subjects is illustrated in figure $2 \mathrm{a}$, where $\log$ the correlation integral $(\mathrm{cm}) \mathrm{r}$ versus $\log \mathrm{r}$ for different embedding dimensions $(\mathrm{m})$ is displayed for respiratory movements. Figure $2 \mathrm{~b}$ shows the slope of the curves from figure $2 \mathrm{a}$, showing a quasi-scaling region (see Appendix). D2 was also calcalated for a phase-randomised surrogate time series generated from the original data using the method of THEILER [16].

Surrogate data analysis. Surrogate data were generated from the original data to construct a linear random time series. To investigate the nonlinearity of the original data, the D2 values a)

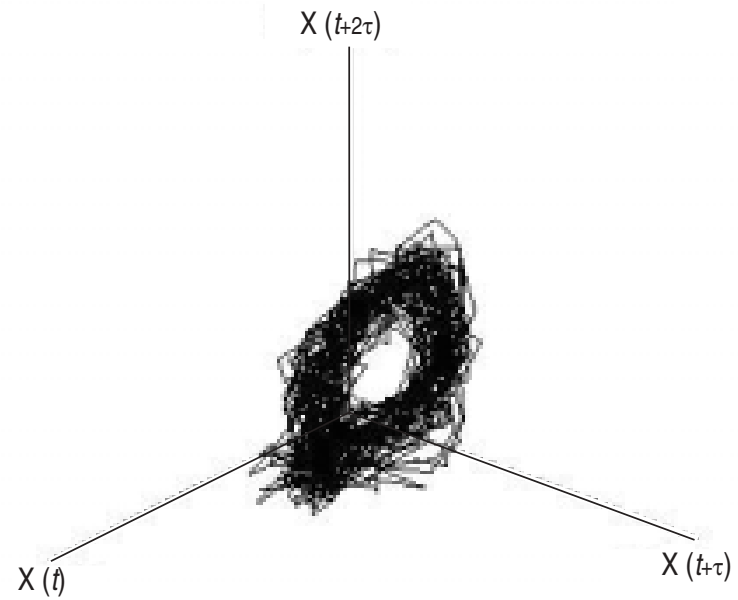

b)

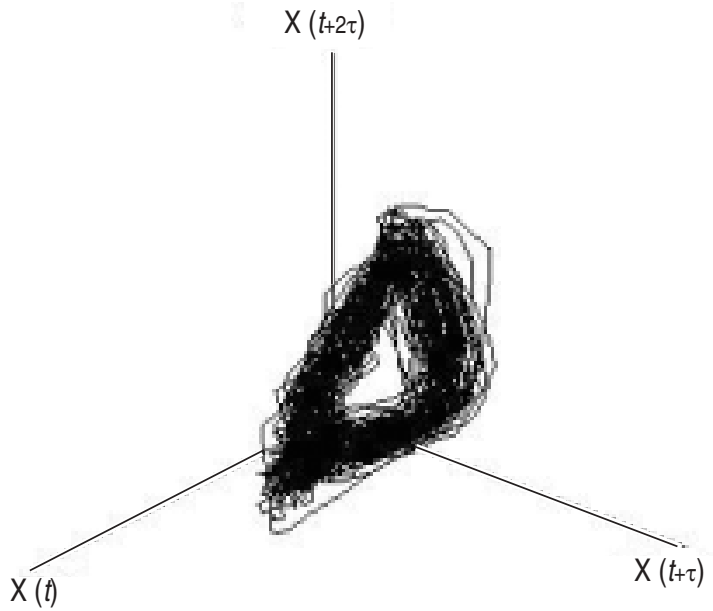

Fig. 1.- Illustrative examples of 3-dimensional attractors. Phase-space representations of respiratory movement in a) control subjects and b) patients with obstructive sleep apnoea/hypopnoea syndrome. Representations were constructed with three-dimensional attractors using Takens' method [16].

obtained with these data were compared with those from surrogate data generated by a linear stochastic process. D2 values of the two time series were compared using the Wilcoxon matched-pairs signed-rank test. A significant difference between the D2 values indicated that the null hypothesis (that the original time series data are linear) can be rejected, meaning that the original data have a nonlinear structure $[17,18]$.

Breath-to-breath variations analysis using conventional methods. In order to estimate breath-to-breath variations, the intervals of 2,000 consecutive artefact-free samples (200 s) were examined for the variations of each in $t \mathrm{I}, t \mathrm{E}$ and $t$ tot. Two data segments were selected, which were used for the analysis of D2. The current authors define $t \mathrm{I}$ as the time between the start of the inspiratory airflow of one breath and the beginning of the expiratory flow of the next breath, $t \mathrm{E}$ as the time between the start of the expiratory airflow of one breath and the beginning of the inspiratory flow of the next breath, and $t$ tot as the total time of $t \mathrm{I}$ and $t \mathrm{E}$ combined. For each factor, the mean, the $\mathrm{SD}$ and the $\mathrm{CV}(\mathrm{CV}=\mathrm{SD} / \mathrm{mean})$ were calculated.

General statistical methods. The values for all of the subjects were estimated and summarised as mean $\pm \mathrm{SD}$. The Wilcoxon 

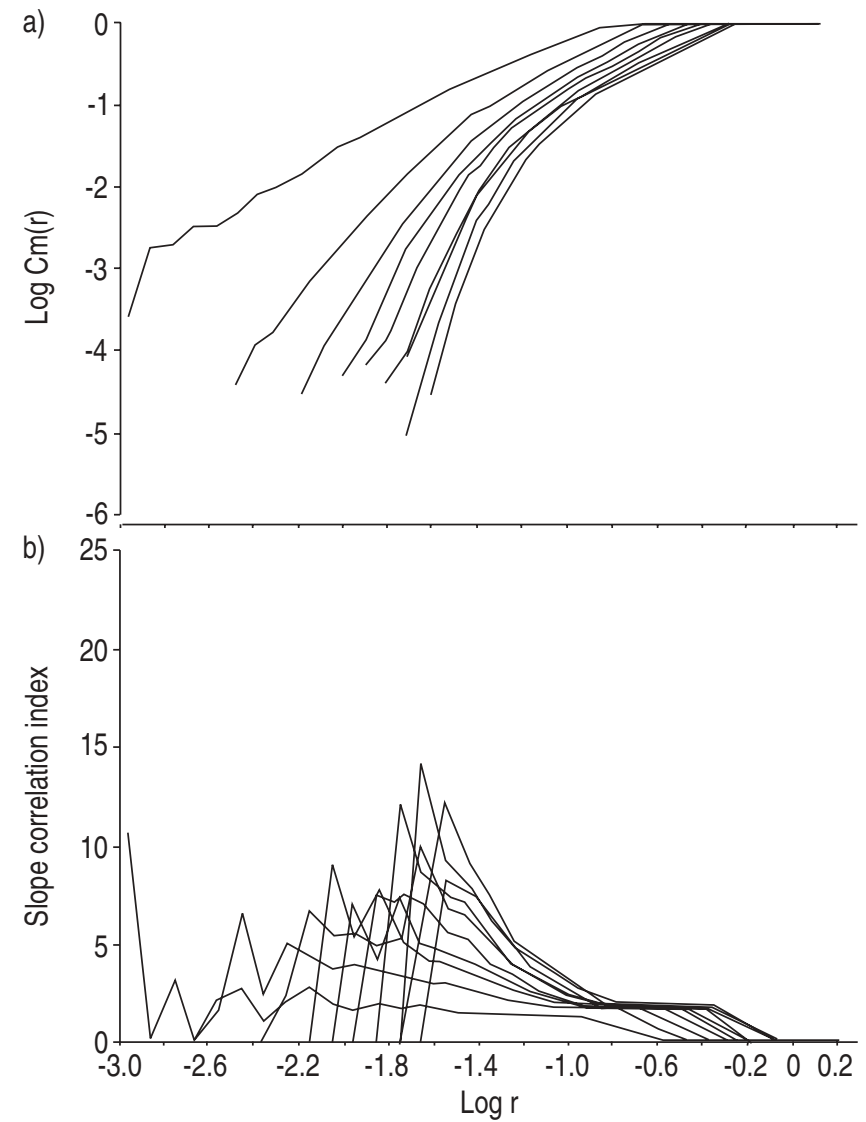

Fig. 2.-One sample of the computation of the correlation dimension during wakefulness. a) $\log \mathrm{Cm}(\mathrm{r})$ versus $\log \mathrm{r}$ of correlation integrals $(\mathrm{m}=2,4,6, \ldots, 20)$, b) the slopes of curves for various embedding dimensions converge to a saturation value of 2.31 , which is the correlation dimension of the attractor in respiration.

matched-pairs signed-rank test (two-sided) was used to compare the D2 values derived from the original and surrogate data (Statview; Abacus Concepts, Inc., Berkeley, $\mathrm{CA}, \mathrm{USA})$. Correlation between the $\mathrm{D} 2$ and the $\mathrm{CV}$ of respiratory movement was calculated using single regression and Pearson's coefficient (Statview; Abacus Concepts). Differences were considered significant at $\mathrm{p}<0.05$.

\section{Results}

During the recording for respiratory movement, it was carefully observed whether the patients fell asleep or remained awake by EEG control. If they fell asleep, the recording was resumed after $3 \mathrm{~min}$ adaptation. The appropriate data of awaking time was $51 \pm 25 \mathrm{~min}$ in patients with OSAHS.

\section{The correlation dimension and breath-to-breath} variations during wakefulness with eyes closed

The values of $\mathrm{D} 2$ for respiratory movement in patients with OSAHS were significantly larger than those in control subjects $(2.49 \pm 0.58$ and $1.76 \pm 0.32$, respectively; $p<0.01)$. On the basis of $t \mathrm{I}, t \mathrm{E}$ and $t$ tot, the mean values and SD showed no significant difference between patients with OSAHS and control subjects. The values of $\mathrm{CV}$ in patients with OSAHS tended to be larger than those in control subjects. Only the $\mathrm{CV}$ of $t \mathrm{E}$ for respiratory movement in patients with OSAHS was significantly larger than that in control subjects $(\mathrm{p}<0.05$; table 1).

\section{Surrogate data analysis}

Table 2 shows the values of D2 from the original data and surrogate data in all subjects. The values of D2 in respiratory movement were statistically significantly larger in the surrogate data than in the original data using the Wilcoxon matched-pairs signed-rank test $(\mathrm{p}<0.01)$.

\section{Relationship between the correlation dimension and coefficient of variation}

D2 for respiratory movement significantly correlated with the CV of $t \mathrm{I}, t \mathrm{E}$ or $t$ tot $(\mathrm{r}=0.50,0.61$ and $0.67 ; \mathrm{p}<0.01$, respectively; fig. 3).

\section{Diagnostic performance of the correlation dimension for respiratory movement}

Diagnostic performance of the D2 for respiratory movement to detect OSAHS is summarised in table 3. All results were statistically significant using Fisher's exact probability test $(p<0.01)$. In the case of $\geqslant 2.0$ of $\mathrm{D} 2$ for respiratory movement, the sensitivity, specificity, positive predictive values and negative predictive values to detect the presence of OSAHS were $85.7 \%, 76.9 \%, 75.0 \%$ and $81.8 \%$, respectively. Receiver operating characteristic (ROC) curve testing was

Table 1.-Correlation dimension (D2) of each breath component during wakefulness with eyes closed in patients with obstructive sleep apnoea/hypopnoea syndrome (OSAHS) and control subjects

\begin{tabular}{lccc}
\hline & Patients with OSAHS & Control subjects & p-value ${ }^{\#}$ \\
\hline Subjects n & 14 & 13 & \\
D2 & $2.49 \pm 0.58$ & $1.76 \pm 0.32$ & $\mathrm{p}<0.01$ \\
$t$ I & $1.61 \pm 0.51$ & $2.16 \pm 1.09$ & \\
$\quad$ Mean & $0.44 \pm 0.21$ & $0.54 \pm 0.22$ & $\mathrm{NS}$ \\
$\quad$ SD & $26.20 \pm 7.81$ & $21.48 \pm 3.43$ & $\mathrm{NS}$ \\
CV & & & \\
$t$ E & $2.31 \pm 0.56$ & $2.91 \pm 1.01$ & $\mathrm{NS}$ \\
Mean & $0.63 \pm 0.31$ & $0.55 \pm 0.33$ & $\mathrm{NS}$ \\
SD & $26.20 \pm 9.56$ & $18.67 \pm 4.10$ & $\mathrm{p}<0.05$ \\
CV & & & \\
$t$ tot & $4.01 \pm 0.91$ & $5.42 \pm 2.09$ & $\mathrm{NS}$ \\
$\quad$ Mean & $0.81 \pm 0.41$ & $0.84 \pm 0.51$ & $\mathrm{NS}$ \\
SD & $19.54 \pm 6.22$ & $15.70 \pm 2.67$ & $\mathrm{NS}$ \\
CV & & & \\
\hline
\end{tabular}

Data presented as mean $\pm \mathrm{SD}$ unless otherwise stated. $t \mathrm{I}$ : inspiratory time; $t$ E: expiratory time; $t$ tot: respiratory cycle time; CV: coefficient variation. \#. calculated by the Wilcoxon matched-paired signed-rank test; NS: nonsignificant.

Table 2. - The correlation dimension (D2) of original and surrogate data for respiratory movement in patients with obstructive sleep apnoea/hypopnea syndrome (OSAHS) and control subjects

Patients with OSAHS Control Subjects

\begin{tabular}{lll}
\hline Subjects n & 14 & 13 \\
Original data & $2.49 \pm 0.58$ & $1.76 \pm 0.32$ \\
Surrogate data & $3.45 \pm 0.61^{* *}$ & $2.85 \pm 0.49^{* *}$ \\
\hline
\end{tabular}

Data are presented as mean \pm SD. D2 was computed as an average of slopes from correlation index at embedding dimensions of 16,18 and 20. D2 of surrogate data was compared with that of original data using the Wilcoxon matched-pairs signed-rank test. ${ }^{* *}: \mathrm{p}<0.01$. 

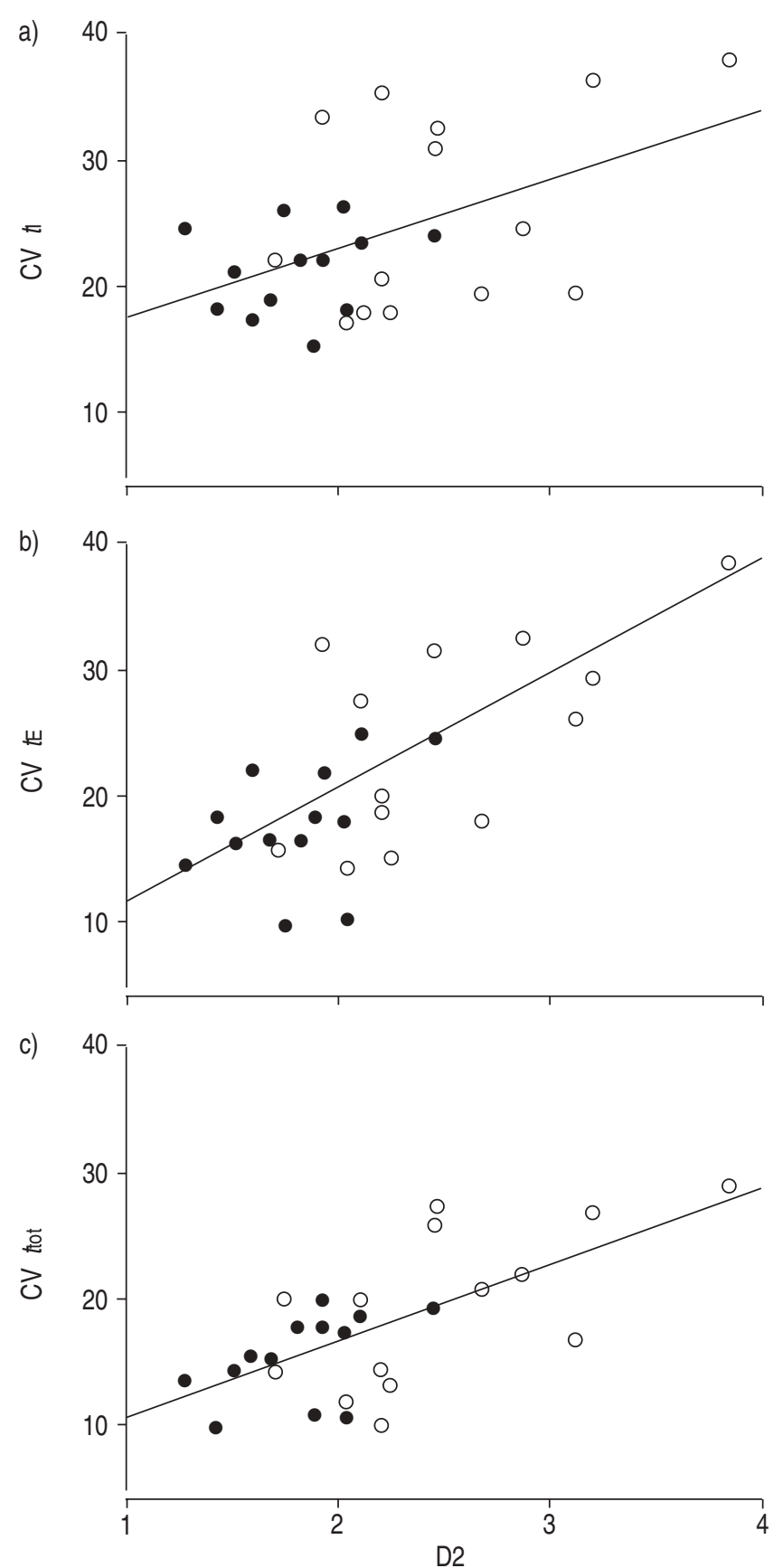

Fig. 3. - The relationship between the coefficient of variation of a) $t$, b) $t \mathrm{E}$ and c) $t$ tot in patients with obstructive sleep apnoea/hypopnoea syndrome $(\bigcirc)$ and control subjects

used to select a cut-off value for detecting the presence of OSAHS.

\section{Discussion}

The present study is the first report investigating dimensionality for respiratory movement and conventional breathto-breath variations during wakefulness with eyes closed in patients with OSAHS. The current study shows the possibility that calculations of $\mathrm{D} 2$, with a brief term respiration data segments ( $3 \mathrm{~min})$, can be used to identify patients with OSAHS.
Table 3.-Diagnostic performance of the correlation dimension (D2) for respiratory movement to detect obstructive sleep apnoea/hypopnoea syndrome (OSAHS)

\begin{tabular}{lcc}
\hline \multirow{2}{*}{ 2 for respiratory movement } & \multicolumn{2}{c}{ AHI $>5 \cdot \mathrm{h}^{-1 \#}$} \\
\cline { 2 - 3 } & Sensitivity \% & Specificity \% \\
\hline$\geqslant 2.2$ & 57.1 & 92.3 \\
$\geqslant 2.1$ & 78.6 & 84.6 \\
$\geqslant 2.0$ & 85.7 & 76.9 \\
$\geqslant 1.9$ & 92.9 & 62.9 \\
$\geqslant 1.8$ & 92.9 & 53.8 \\
$\geqslant 1.7$ & 100.0 & 46.2 \\
\hline
\end{tabular}

AHI: apnoea-plus-hypopnoea index. ${ }^{*}$ : $n=14$.

\section{Analysis of the dimensionality for respiratory movement}

The study of the dynamics of nonlinear systems that can display not only regular behaviour but also disordered turbulent behaviour has pioneered new ways to characterise the signal properties of biological processes. Nonlinear systems with fractal dynamics (e.g. the neuroautonomic mechanisms regulating heart rate variability) behave as if they are driven far from equilibrium under basal conditions. A kind of complex variability, rather than a single homoeostatic steady state, seems to define the free running function of many biological systems [19]. Recently, techniques from nonlinear dynamical systems analysis have been widely applied to EEG, heart rate variability and respiration. In order to make clear problems related to the nature of EEG, heart rate variability and respiratory movement, as well as to discriminate between different physiological states, D2 measurements have often been calculated. However, a small number of nonlinear dimensional analyses of respiration have been reported previously. Bock et al. [20] reported that, by analysing the largest Lyapunov exponent and correlation dimension, the respiratory state in sleeping patients with apnoea could be shown to have chaotic dynamics. The current authors have previously shown that respiratory movement in patients with severe OSAHS during apneoic sleep was random, as no D2 for respiratory movement during sleep with apnoea could be obtained [13]. In the present study, D2 for the respiratory movement in patients with OSAHS and in control subjects was calculated during wakefulness with eyes closed. The current authors suggest that patients with OSAHS have abnormalities in waking respiration. Further studies are needed to investigate the cause of the respiratory abnormalities during wakefulness.

\section{Comparison of the original and surrogate data}

Precise interpretation of the D2 statistics can be problematic. THEILER et al. [17] developed the concept of "surrogate data", a method that provides a way to test specific null hypotheses by comparing D2 values from original data and appropriately constructed surrogate data. To test whether nonlinear dynamics characterised the time series of respiratory movement, surrogate data were generated for a comparison of D2 from the surrogate data with that from the original data. A clearly different result for the original data than for the surrogate data would indicate that the null hypothesis (i.e. a linear process) could be rejected. In the present study, statistically significant differences in D2 $(p<0.05)$ were found between the original and surrogate data, with D2 being larger in the surrogate data. These results indicate that respiratory movements in wakefulness with eyes 
closed have nonlinear deterministic properties in both control subjects and patients with OSAHS.

\section{Analysis of conventional breath-to-breath variations}

On a breath-to-breath basis, assessment of the stability of the respiratory pattern generally proceeds from a measurement of the usual respiratory variables (e.g. $V \mathrm{~T}, t \mathrm{I}, t \mathrm{E}$ and $t$ tot). The SD or CV have been utilised as the measure of variability of the respiratory pattern. During waking respiration, the CV of the breath-to-breath values of $V \mathrm{~T}$ and $t \mathrm{I}$ and $t \mathrm{E}$ are typically $10-30 \%$ in a steady state [21]. In this study, $t \mathrm{I}$, $t \mathrm{E}$ and $t$ tot (mean, SD and CV) were examined. $V \mathrm{~T}$ could not be measured in the present systems. The obtained data were similar to those in previous reports [22, 23]. A significant reduction in $t \mathrm{E}$ was observed in obese subjects [22]. To make an exception of the factor of obesity, the authors recruited BMI-matched control subjects. CARLEY et al. [24] examined the interactions between $t \mathrm{I}, t \mathrm{E}$ and inspiratory oesophageal pressure generation in seven subjects with occlusive sleep apnoea. Significant breath-to-breath oscillations occur in $t \mathrm{I}$, $t \mathrm{E}$ and inspiratory oesophageal pressure during the repetitive apnoeas of sleep apnoea syndrome. Negative synchronisation of $t \mathrm{I}$ and $t \mathrm{E}$ may contribute to periodic upper airway collapse [24]. In regard to competition with control subjects, only CV ( $t$ E) was significantly larger in the current study's patients. Furthermore, the current authors first demonstrated a relationship between $\mathrm{D} 2$ and $\mathrm{CV}$ ( $t \mathrm{I}, t \mathrm{E}$ and $t$ tot) during wakefulness with eyes closed $(\mathrm{p}<0.01)$. This might suggest that the complexity of respiratory movement increases in patients with OSAHS during wakefulness with eyes closed.

\section{Comparison of patients with OSAHS and control subjects}

Compared with control subjects, D2 for respiratory movement was significantly larger in patients with OSAHS during wakefulness with eyes closed $(1.76 \pm 0.32$ and $2.49 \pm 0.58$, respectively). The current authors' previous study showed a reduction of complexity by using nasal continuous positive airway pressure in patients with OSAHS [13]. In the present study, the finding of a significant increase in dimensionality in patients with OSAHS can be taken to show an increase in the complexity and a decrease in the regularity of the respiration.

It is useful to estimate pathological state nonlinear analysis [25-27]. The irregular ventricular response in atrial fibrillation or ventricular fibrillation itself represents deterministic cardiac chaos [28]. The subtle but complex heart-rate fluctuations seen during normal sinus rhythm in healthy individuals are attributable in part to deterministic chaos, and various diseases, such as congestive heart failure syndrome, may involve a paradoxical decrease of nonlinear variability [25]. The beat-to-beat alternation in the QRS axis and in amplitude were observed in some cases of cardiac tamponade [26]. In this study, the authors suggested that the analysis of D2 for respiratory movement during daytime wakefulness was a useful and objective screening method to distinguish patients with OSAHS from normal subjects.

In summary, the current authors speculate that recordings of respiratory movement with a brief term make it possible to objectively detect the presence of the sleep-related breathing disorders before diagnostic polysomnography. However, prospective evaluation is required to put this method on a firm basis. Its suitability at the primary care level remains to be determined.

\section{Appendix}

\section{The Grassberger-Procaccia algorithm}

The Grassberger-Procaccia algorithm is the method for quantifying the dimensionality of attractors from biological time series. This algorithm attempts to determine the dimension of the attractor of a nonlinear dynamical system. The correlation integral $\mathrm{Cm}(\mathrm{r})$ is defined by the following equation:

$$
\mathrm{Cm}(\mathrm{r})=1 / \mathrm{N}(\mathrm{N}-1) \sum_{\mathrm{i}=1}^{\mathrm{N}} \sum_{\mathrm{j}=1}^{\mathrm{N}} \Theta\left(\mathrm{r}-\left|\mathrm{X}_{\mathrm{i}}-\mathrm{X}_{\mathrm{j}}\right|\right), \mathrm{i} \neq \mathrm{j}
$$

where $\Theta(\mathrm{t})$ is the Heaviside function $(\Theta(\mathrm{t})=1$ if $t \geqslant 0, \Theta(\mathrm{t})=0$ if $t<0)$; the time series is $\times(t \mathrm{i})$, with $\mathrm{i}=1,2,3, \ldots$; the vector $\mathrm{X}_{\mathrm{i}}$ is $\{\mathrm{x}(t \mathrm{i}), \quad \mathrm{x}(t \mathrm{i}+\tau), \quad \ldots, \quad \mathrm{x}(t \mathrm{i}+(\mathrm{m}-1) \tau)\}$, with $|\mathrm{Xi}-\mathrm{Xj}|$ being the Euclidean distance between vector $\mathrm{Xi}$ and $\mathrm{Xj} ; \tau$ is time lag; and $\mathrm{m}$ is embedding dimension. $\mathrm{Cm}(\mathrm{r})$ behaves as a power law of $r$; that is:

$$
\begin{gathered}
\mathrm{Cm}(\mathrm{r}) \propto \mathrm{r}^{v} \\
\log \mathrm{Cm}(\mathrm{r}) \propto v \log \mathrm{r}
\end{gathered}
$$

where $v$ is the correlation index meaning the slope of the curve of $\log \mathrm{Cm}(\mathrm{r})$ versus $\log \mathrm{r}$. When the values of the correlation index in the embedding dimension of 16-20 plateaued in the original data, the values of the correlation index from embedding dimensions 16-20 were averaged to estimate a definite value of the D2 in this study. A necessary condition for the computation of dimensionality is the construction of the phase space for the analysis of experimental data, usually using the method of TAKENS [15], which spans the phase space by the time-shift method. The concept of the phase space is central to the analysis of nonlinear dynamics [29]. This means that an m-dimensional phase space was spanned by $\{\times(t)$, $\times(t+\tau), \ldots, \times[t+(\mathrm{m}-1) \tau]\}$. The time lag $\tau$ was selected by finding the first lag that reduced the value of the autocorrelation function to $1 / \mathrm{e}$ of its initial value [30]. The respiratory signals were embedded into phase spaces that increased by an embedding dimension of 2-20. After embedding the signals, the correlation integral $(\mathrm{Cm}(\mathrm{r})$ for distance $\mathrm{r})$ was calculated using the Grassberger-Procaccia algorithm (fig. 2a) [31]. The slopes of these curves then were plotted against $\log r$ (fig. 2b). The limits of the quasi-linear region were determined by a display of slope (correlation index) versus log $\mathrm{r}$ curves [30, 32]. The scaling region had to be linear, and the length that was adopted for the scaling region in the original data in this study was 0.4. When the values of the correlation index in the embedding dimension of 16-20 defined a plateau, these values were averaged to estimate D2. The correlation index is defined as the slope in the range of the scaling region on the plot of $\log \mathrm{Cm}(\mathrm{r})$ versus $\log \mathrm{r}$ by using the least squares fit through the points $[12,13,33]$. If a portion of the surrogate data did not define a plateau, the same scaling region used for the original data (length, 0.4) was adopted, and the mean correlation index was used from the embedding dimension of 16- 20 as D2 for comparison with D2 in the original data.

\section{References}

1. The report of an American Academy of Sleep Medicine Task Force. Sleep-related breathing disorders in adults: recommendations for syndrome definition and measurement techniques in clinical research. Sleep 1999; 22: 667-689.

2. Johns MW. A new method for measuring daytime sleepiness: the Epworth sleepiness scale. Sleep 1991; 14: 540-545. 
3. Carskadon MA, Dement WC. The multiple sleep latency test: what does it measure? Sleep 1985; 5: Suppl. 2, 67-72.

4. Priban IP. An analysis of some short-term patterns of breathing in man at rest. $J$ Physiol 1963; 166: 425-434.

5. Winning AJ, Hamilton RD, Shea SA, Knott C, Guz A. The effect of airway anaesthesia on the control of breathing and the sensation of breathlessness in man. Clin Sci 1985; 68: 215-225

6. Tobin MJ, Mador M, Guenther S, Lodato RF, Sackner MA. Variability of resting drive and timing in healthy subjects. J Appl Physiol 1988; 65: 309-317.

7. Kowallik P, Jacobi I, Jirmann A, Meesmann M, Schmidt M, Wirtz H. Breath-to-breath variability correlates with apneahypopnea index in obstructive sleep apnea. Chest 2001; 119: 451-459.

8. Achermann P, Hartmann R, Gunzinger A, Guggenbuhl W, Borbely AA. Correlation dimension of the human sleep electroencephalogram: cyclic changes in the course of the night. Eur J Neurosci 1994; 6: 497-500.

9. Kaplan DT, Furman MI, Pincus SM, Ryan SM, Lipsitz LA, Goldberger AL. Aging and the complexity of cardiovascular dynamics. Biophys $J$ 1991; 59: 945-949.

10. Small M, Judd K, Lowe M, Stick S. Is breathing in infants chaotic? Dimension estimates for respiratory patterns during quiet sleep. J Appl Physiol 1999; 86: 359-376.

11. Burioka N, Cornelissen G, Halberg F, Kaplan DT. Relationship between correlation dimension and indices of linear analysis in both respiratory movement and electroencephalogram. Clin Neurophysiol 2001; 112: 1147-1153.

12. Sako T, Burioka N, Suyama H, Nomura T, Takeshima T, Shimizu E. Nonlinear behavior of human respiratory movement during different sleep stages. Chronobiol Int 2001; 18: 71-83.

13. Miyata M, Burioka N, Suyama H, et al. Non-linear behaviour of respiratory movement in obstructive sleep apnoea syndrome. Clin Physiol Funct Imaging 2002; 22: 320-327.

14. Eckmann JP, Ruelle D. Fundamental limitations for estimating dimension and Lyapunov exponents in dynamical systems. Physica D 1992; 56: 185-187.

15. Takens F. Detecting strange attractors in turbulence. In: Rand D, Young BS, ed. Lecture Notes in Mathematics. Berlin, Springer, 1981; pp. 366-381.

16. Theiler J. Estimating fractal dimension. J Opt Soc Am A 1990; 7: 1055-1073.

17. Theiler J, Eubank S, Longtin A, Galdrikian B, Farmer JD. Testing for nonlinearity in time series: the method of surrogate data. Physica D 1992; 58: 77-94.

18. Schreiber T, Schmitz A. Improved surrogate data for nonlinearity tests. Phys Rev Lett 1996; 77: 635-638.
19. Goldberger AL. Non-linear dynamics for clinicians: chaos theory, fractals, and complexity at the bedside. Lancet 1996; 347: $1312-1314$

20. Bock J, Gough DA. Toward Prediction of Physiological State Signals in Sleep Apnea. IEEE Trans Biomed Eng 1998; 45: 1332-1341.

21. Bruce EN, Daubenspeck JA. Mechanism and analysis of ventilatory stability. In: Dempsey JA, Pack AI, ed. Lung Biology in Health and Disease, Volume 79: Regulation of Breathing. New York, Marcel Dekker, 1995; pp. 285-313

22. Burki NK, Richard WB. Ventilatory regulation in eucapnic morbid obesity. Am Rev Respir Dis 1984; 129: 538-543.

23. Tobin MJ, Yang KL, Jubran A, Lodato RF. Interrelationship of breath components in neighboring breaths of normal subjects. Am J Respir Crit Care Med 1995; 152: 1967-1976.

24. Carley DW, Onal E, Aronson R, Lopata M. Breath-bybreath interactions between inspiratory and expiratory duration in occlusive sleep apnea. J Appl Physiol 1989; 66: 2312-2319.

25. Goldberger AL, Rigney DR, Mietus J, Antman EM, Greenwald S. Nonlinear dynamics in sudden cardiac death syndrome: heart rate oscillations and bifurcations. Experientia 1988; 44: 983-987.

26. Rigney DR, Goldberger AL. Nonlinear mechanics of the hearts swinging during pericardial effusion. Am J Physiol 1989; 257: H 1292-1305.

27. Rosenbaum DS, Jackson LE, Smith JM, Garan H, Ruskin JN, Cohen RJ. Electrical alternans and vulnerability to ventricular arrhythmia. N Engl J Med 1994; 330: 235-241.

28. Peng CK, Havlin S, Stanley HE, Goldberger AL. Quantification of scaling exponents and crossover phenomena in nonstationary heartbeat time series. Chaos 1995; 5: 82-87.

29. Kaplan D. Self-similarity and fractal geometry. In: Understanding Nonlinear Dynamics. Kaplan D, Leon G, eds. New York, Springer-Verlag, 1995; pp. 104-145.

30. Albano AM, Muench J, Schwartz C, Mess AI, Rapp PE. Singular-value decomposition and the Grassberger-Procaccia algorithm. Phys Rev Lett 1988; 38: 3017-3026.

31. Grassberger P, Procaccia I. Characterization of strange attractors. Phys Rev Lett 1983; 50: 346-349.

32. Lehnertz K, Elger CE. Spatio-temporal dynamics of the primary epileptogenic area in temporal lobe epilepsy characterized by neuronal complexity loss. Electroencephalogr. Clin Neurophysiol 1995; 95: 108-117.

33. Cutler CD. Some results on the behavior and estimation of the fractal dimensions of distributions on attractors. $J$ Stat Phys 1991; 62: 651-708. 